\title{
BMJ Open mCARE, a digital health intervention package on pregnancy surveillance and care-seeking reminders from 2018 to 2027 in Bangladesh: a model-based cost- effectiveness analysis
}

To cite: Jo Y, LeFevre AE, Ali $\mathrm{H}$, et al. mCARE, a digital health intervention package on pregnancy surveillance and care-seeking reminders from 2018 to 2027 in Bangladesh: a model-based cost-effectiveness analysis. BMJ Open 2021;11:e042553. doi:10.1136/ bmjopen-2020-042553

- Prepublication history and supplemental material for this paper is available online. To view these files, please visit the journal online (http://dx.doi. org/10.1136/bmjopen-2020042553).

Received 08 July 2020 Revised 24 February 2021 Accepted 25 February 2021

Check for updates

(C) Author(s) (or their employer(s)) 2021. Re-use permitted under CC BY-NC. No commercial re-use. See rights and permissions. Published by BMJ.

${ }^{1}$ Johns Hopkins Bloomberg School of Public Health, Baltimore, Maryland, USA 2JiVitA program, Johns Hopkins Bloomberg School of Public Health, Rangpur, Bangladesh ${ }^{3}$ mPOWER Social Enterprises, Dhaka, Bangladesh

Correspondence to

Dr Youngji Jo;

youngji1435@gmail.com

\section{ABSTRACT}

Objective We estimated the cost-effectiveness of a digital health intervention package (mCARE) for community health workers, on pregnancy surveillance and care-seeking reminders compared with the existing paper-based status quo, from 2018 to 2027, in Bangladesh.

Interventions The mCARE programme involved digitally enhanced pregnancy surveillance, individually targeted text messages and in-person home-visit to pregnant women for care-seeking reminders for antenatal care, child delivery and postnatal care.

Study design We developed a model to project population and service coverage increases with annual geographical expansion (from 1 million to 10 million population over 10 years) of the mCARE programme and the status quo. Major outcomes For this modelling study, we used Lives Saved Tool to estimate the number of deaths and disability-adjusted life years (DALYs) that would be averted by 2027, if the coverage of health interventions was increased in mCARE programme and the status quo, respectively. Economic costs were captured from a societal perspective using an ingredients approach and expressed in 2018 US dollars. Probabilistic sensitivity analysis was undertaken to account for parameter uncertainties.

Results We estimated the mCARE programme to avert 3076 deaths by 2027 at an incremental cost of $\$ 43$ million relative to the status quo, which is translated to $\$ 462$ per DALY averted. The societal costs were estimated to be $\$ 115$ million for mCARE programme ( $48 \%$ of which are programme costs, $35 \%$ user costs and $17 \%$ provider costs). With the continued implementation and geographical scaling-up, the mCARE programme improved its cost-effectiveness from $\$ 1152$ to $\$ 462$ per DALY averted from 5 to 10 years.

Conclusion Mobile phone-based pregnancy surveillance systems with individually scheduled text messages and home-visit reminder strategies can be highly cost-effective in Bangladesh. The cost-effectiveness may improve as it promotes facility-based child delivery and achieves greater programme cost efficiency with programme scale and sustainability.

\section{Strengths and limitations of this study}

- From a societal perspective, we assessed the costeffectiveness of a digital health intervention on pregnancy surveillance and care-seeking promotion relative to the paper-based status quo with incremental geographical scale-up in Bangladesh.

- Given the limited empirical data and existing practice of a large scaled-up mHealth programme in pregnancy surveillance and care-seeking reminders, we used estimates drawn from observed data and a range of assumptions regarding the population and service coverage metrics using Lives Saved Tool to forecast health impact and costs.

- We identified the key drivers and enabling conditions of cost-effectiveness through various sensitivity analyses and demonstrated the quantitative benefits of continued implementation and geographical expansion that improves the programme cost-effectiveness over time at scale.

- This finding extends earlier study with detailed activity lists and cost estimates under a government-led scale-up scenario to help implementation planning and real-world investment decision-making.

\section{INTRODUCTION}

Bangladesh is the eighth most populous country in the world with 165 million people, and accounts a maternal mortality ratio of 173 per 100000 live births, ${ }^{1} 74000$ newborn deaths ${ }^{2}$ and 83000 stillbirths $^{3}$ each year. Many maternal and neonatal deaths can be averted using evidence-based interventions such as use of antenatal care (ANC) and timely access to healthcare. In many low-income and middle-income countries, however, coverage levels of these interventions are still low. Despite the substantial progress over the last few decades, Bangladesh still has the lowest coverage of these interventions (eg, 
$21 \%$ of pregnant women attend at least four ANC visits and $31 \%$ have skilled birth attendants) among its South Asian neighbours. ${ }^{45}$ Improving maternal and newborn health $(\mathrm{MNH})$ remains an essential health priority for Bangladesh. ${ }^{6}$

Over the recent decade, mHealth (ie, defined as use of mobile and wireless technologies to support the achievement of health objectives $)^{7}$ has been widely used to improve health outcomes, service access and quality of essential MNH services. ${ }^{8}{ }^{9}$ Several studies demonstrated the positive impact of mHealth in resource-limited settings. ${ }^{1011}$ These included an increase in the use of ANC from $10 \%$ to $37 \%$ in southern India, ${ }^{12}$ four or more ANC visits (OR 3.21, 95\% CI 1.73 to 5.98) in South Africa ${ }^{13}$ after introduction of text messages for health promotion, and a significant increase in the coverage of skilled birth attendants $(11 \%)$, facility delivery $(10 \%)$ and measles immunisation $(6 \%)$ in Ghana. ${ }^{14}$ Use of mHealth also increased knowledge retention of health workers about the identification of danger signs during pregnancy, after delivery and among newborns and children in lowincome and middle-income countries. ${ }^{15}$

Under these circumstances, a wide array of digital health solutions have been piloted throughout the last few decades in Bangladesh, ${ }^{16}$ including telemedicine, short message service (SMS) advice for safe pregnancy and use of the mobile phone as a data collection tool ${ }^{17}$ to improve access and quality of service delivery of essential MNH services. ${ }^{18}$ However, few of these successfully piloted innovations have been scaled nationwide sustainably. ${ }^{19-21}$ Studies acknowledge that this persistent challenge is partly due to a lack of evidence on the value for money of mHealth programmes in MNH services, how to build capacity in countries and what resources are needed. ${ }^{22}$

The mCARE-I research project, implemented from 2011 to 2015, tested a package of mHealth interventions to support community health workers (CHWs) on pregnancy surveillance activities and to promote careseeking behaviours to pregnant women and their families in the Gaibandha district in northern Bangladesh. A previous cost-effectiveness study estimated that using SMS reminders for ANC care-seeking was highly cost-effective with an estimate of $\$ 31$ per disability-adjusted life year (DALY) averted. ${ }^{23}$ To further promote a large scale-up of the promising intervention, the objective of this study is to assess the cost-effectiveness of the mCARE programme, compared with the current paper-based status quo, over a 10-year analytic time horizon (2018-2027) to help guide investment decisions and to promote large-scale use and sustainability of mHealth intervention.

\section{METHODS}

\section{Study setting}

Gaibandha district is one of the 64 districts in Bangladesh which consists of approximately 15.8 million people (6249 square miles). The coverage for $\mathrm{MNH}$ services is $31 \%$ for four or more ANC visits, $38 \%$ for facility delivery and $36 \%$ for postnatal care (PNC), which compares favourably with national estimates. ${ }^{24}$ The literacy rate is $46 \%$ in Gaibandha, ${ }^{25}$ which are lower than the national literacy rate as $75 \%$ and primary education completion rate as $68 \%$ in Bangladesh. ${ }^{26}$

\section{Intervention}

In this analysis, we consider three comparators and their associated programmatic activities: (1) the comprehensive mCARE programme involves a digital intervention package with both supply-side and demand-side promotion components. This includes pregnancy surveillance using a mobile phone-based system by government CHWs (family welfare assistants) and automated SMS and CHW home-visit reminders to pregnant women sent before individually scheduled ANC 1-4 dates; (2) the basic mCARE package involves only on the supply-side promotion component which is pregnancy surveillance using a mobile phone-based system by the government CHWs; and (3) the status quo involves paper-based pregnancy surveillance and existing community-based ANC promotion activities to pregnant women by the government CHWs.

\section{Model}

We developed a model that can estimate the annual target population as the number of pregnant women, population coverage (as the number of pregnant women who were registered in the programme through census enumeration and pregnancy surveillance) and service coverage (as the number of pregnant women who sought care, over the number of registered pregnant women in the system) from 2018 to 2027 (online supplemental appendix figure S1). For simplicity, we assumed a hypothetical 1 million population per district and incremental scale-up of the intervention to additional districts each year for upto 10 districts over a 10-year time horizon (ie, step-wedge scale-up). For a target population (ie, pregnant women), the number of pregnant women per district ${ }^{27}$ was calculated based on the proportional ratio of number of married women of reproductive age (MWRA), aged $15-49$ years, in Gaibandha district $(24 \%),{ }^{28}$ fertility rate (ie, 66 births per 1000 MWRA), ${ }^{29}$ abortion rate (ie, 29 per 1000 pregnant women) ${ }^{30}$ and fetal loss rate (ie, 28 per 1000 births) ${ }^{31}$ Similarly, we estimated the number of CHWs per district based on the average ratio (ie, 1 CHW for identifying and managing about 30 pregnant women per year in their catchment area) that we obtained from the interview with local public health managers and reviewed by local experts in Gaibandha district (online supplemental appendix tables S1 and S2).

\section{Population coverage}

As a measure of workforce performance efficiency in pregnancy surveillance, emphasis is placed on to what extent mCARE system can improve existing productivity and operational efficiency compared with paper-based system. We focused on the incremental increases in the 
number of household visits per day by a CHW within a feasible range of the CHW's existing capacity (eg, an average 30 household visits per CHW per day). With improved workforce efficiency, we assumed the mCARE system would enrol $90 \%$ of the target population (total number of pregnant women) through census enumeration and pregnancy surveillance, while the paper-based system would capture $80 \%$ of the target population over the year in each district based on the interviews with relevant local stakeholders (table 1).

\section{Service coverage}

Service coverage represents a measure of the pregnant woman's care-seeking to essential maternal and newborn care services during pregnancy, childbirth and postnatal periods. Until further data become available, we took the assumptions of the annual coverage increase rate for each scenario from baseline to the target year as plausible estimates for expansion: $10 \%$ for ANC and PNC and 5\% for child delivery for the comprehensive mCARE programme, $5 \%$ and $2.5 \%$ for the basic mCARE programme, and $1 \%$ and $0.5 \%$ for the paper-system, respectively, based on empirical and published data. ${ }^{32}$ For example, the trends of ANC coverage from the Bangladesh Demographic and Health Survey revealed an increase from 58\% in 2004 to $88 \%$ in 2014 with a $3 \%-5 \%$ annual coverage increase rate. ${ }^{24}$ A quasi-experimental evaluation of the pilot project suggests that the comprehensive mCARE programme was associated with a 2.6 times increase in the use of ANC and a 1.6 times increase in $\mathrm{PNC}^{32}$ compared with the baseline. Consequently, our coverage increase rates assumption similarly resulted in a 2.4 times coverage increase of ANC and PNC using the comprehensive mCARE programme, 1.6 times increase with the basic mCARE programme and 1.1 times increase in coverage for the status quo, from the respective baseline values in 2018 to 2027. Since child delivery service is the most resource-intensive care, the annual increase rates of skilled birth attendance and facility delivery were adjusted to half of ANC and PNC services increase rates to account for limited health system capacity (online supplemental appendix table S3).

\section{Health outcome}

The Lives Saved Tool (LiST) V.5.761 ${ }^{33}$ was then used to generate estimates of the incremental numbers of lives saved based on the respective incremental service coverage increases for ANC, child delivery and PNC for each scenario (online supplemental appendix table S3). We did not include immunisation as the coverage levels were already high $(>95 \%)$ in Bangladesh in 2018. Based on country-wide demographics, efficacy and coverage data, ${ }^{33}$ LiST produced projections of the annual number of lives saved for mothers, stillbirths and newborns from 2018 to 2027. Considering the incremental geographical expansion over time, we proportionally adjusted the annual number of lives saved based on the increased target population size in the given year implemented by each scenario. We calculated DALYs based on the number of lives saved ${ }^{29} 3435$ and the life table in Bangladesh, ${ }^{36}$ assuming life expectancy as 72 years for the newborn and 30 years for mothers with an annual $3 \%$ discount rate (resulting in 27 DALYs per mother's death and 30 DALYs per newborn/stillbirth death). Due to the lack of morbidity data, years lived with disability were not included in the DALY calculation and were expected to make a marginal change to the total DALY measure. ${ }^{37}$

\section{Societal costs}

Economic costs were measured from a societal perspective including programmes, health systems and user costs over the 10-year time horizon. ${ }^{38}$ We estimated programme costs based on an ingredients approach. For each scenario, we identified detailed activity lists (eg, system optimisation, training and implementation) and collected relevant cost information (eg, salary and level of effort for activities) from the interview with government health providers and local experts in the Gaibandha district ${ }^{23}$ (online supplemental appendix tables S1 and S2) and the previously published study ${ }^{23}$ based on the mCARE-1 pilot study from 2012 to 2015. These costs were combined with relevant estimates of number of pregnant women, population coverage and service coverage for each scenario to calculate total programme, provider and user costs (tables 1 and 2). We assumed certain activity and capital costs such as system optimisation to occur only in the first year one time for all districts for the mCARE programme, while census enumeration to occur only in the first year in each district in both the mCARE programme and the status quo. All other activities and associated costs were accounted for each year and each district with annual geographical expansion. All costs were adjusted to 2018 US dollars based on gross domestic product (GDP) deflator, discounted at an annual rate of $3 \%,{ }^{39}$ and capital costs were annualised based on their life expectancy.

We estimated health system costs based on the provider's salary and time consumed for providing a service, as well as commodity consumption (eg, micronutrients or vitamin A) stemming from the use of health services for ANC, child delivery and PNC. Costs to expand or upgrade infrastructure related to these interventions were not included. We calculated user costs including direct and indirect costs for seeking care during ANC, facility or home deliveries and PNC. Direct user costs were any expenses spent to seek care, including round-trip transportation costs, fees for service, medical tests or drugs. Indirect user costs were calculated from opportunity costs that were foregone due to care-seeking such as wages lost by care-seeking from the total time of round-trip transportation, waiting, consultation and treatment. These data were obtained from a previously published study ${ }^{40}$ based on the direct observation study and exit interviews with pregnant women and consultation with government health workers and local experts. 
Table 1 Summary of model parameters and uncertainties for probabilistic sensitivity analysis

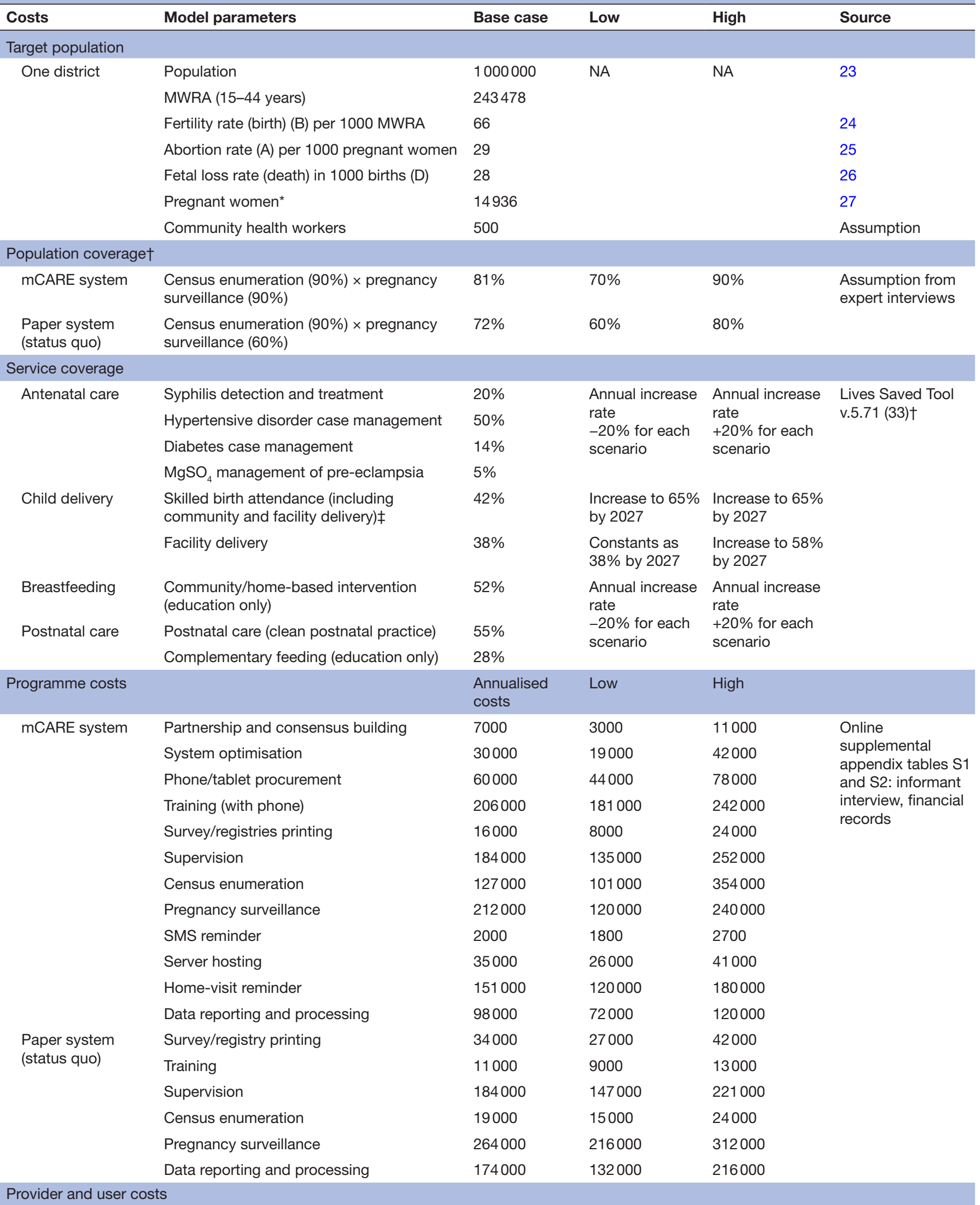




\begin{tabular}{|c|c|c|c|c|c|}
\hline Costs & Model parameters & Base case & Low & High & Source \\
\hline Antenatal care & Provider unit cost & 2.47 & 1.95 & 2.99 & 40 \\
\hline \multirow[t]{2}{*}{ Home delivery } & Provider unit cost & 5.50 & 4.00 & 7.00 & \\
\hline & User unit costs & 19.00 & 3.00 & 35.00 & \\
\hline Facility delivery & User unit costs & 79.00 & 11.00 & 147.00 & \\
\hline \multirow[t]{2}{*}{ Postnatal care } & Provider unit cost & 1.23 & 0.62 & 1.83 & \\
\hline & User unit costs & 7.05 & 0.26 & 13.83 & \\
\hline
\end{tabular}

${ }^{*}$ Number of pregnant women $=$ MWRA $/ 1000 \times\{(\mathrm{B} \times \mathrm{Pb})+(\mathrm{A} \times \mathrm{Pa})+(\mathrm{D} \times \mathrm{Pd})\}$, where $\mathrm{P}$ is the proportion of the year a woman is pregnant for each pregnancy outcome by month ( $\mathrm{Pb}: 9$ months $=0.75, \mathrm{~Pa}: 2$ months $=0.167, \mathrm{Pd}: 3$ months $=0.25)$. (27)

†Coverage data for this indicator are drawn from Demographic and Health Survey, Multiple Indicator Cluster Survey and other nationally representative household surveys. ${ }^{28}$

$\ddagger$ Percent of children born with a skilled attendant present, including doctors, nurses or midwives, in a facility or at home/community. MWRA, married women of reproductive age; NA, not applicable; SMS, short message service.

\section{Sensitivity analyses}

To test the robustness of our cost-effectiveness outcome estimates between the comprehensive mCARE system and the status quo, we performed a series of sensitivity analyses (one-way, three-way and probabilistic sensitivity analysis (PSA)). Parameter uncertainty ranges were determined based on primary data and financial records for the costs, and informant interviews for the service coverage parameters (table 1). The three-way sensitivity analysis was performed based on the three most influential parameters identified by the one-way sensitivity analysis. For PSA, all model parameter values were randomly sampled over 10000 iterative Monte Carlo simulations based on pre-specified distributions of each data parameter to generate $95 \%$ uncertainty ranges around our primary cost-effectiveness estimate. The incremental cost-effectiveness ratio (ICER) estimates were evaluated against different willingness-to-pay thresholds representing a range of financial and budgetary constraints in Bangladesh for public health interventions (eg, GDP per capita). ${ }^{41}$

\section{Patient and public involvement}

Patients or the public were not involved in the design, or conduct, or reporting or dissemination plans of our research.

\section{RESULTS \\ Costs}

Our model estimated that the comprehensive mCARE programme would require a total of $\$ 115$ million by 2027 (including $\$ 55$ million in programme costs, $\$ 40$ million in user costs and $\$ 20$ million in provider costs), which is an increment of $\$ 19$ million, compared with the mCARE basic programme, and $\$ 43$ million compared with the status quo. In the comprehensive mCARE programme, the major programme cost makeup consisted of pregnancy surveillance (19\%), training (18\%) and supervision $(16 \%)$ (table 1$)$.

\section{Effectiveness}

With annual service coverage increase with geographical expansion over 10 years, a total of 3324 estimated lives (uncertainty range (UR): 1777-4871) would be saved, including 1745 neonatal deaths (UR: 1040-2451), 1451 stillbirths (UR: 683-2219) and 127 maternal deaths (UR: 54-201) in the comprehensive mCARE programme. This is about two times greater than the basic mCARE programme (1361 lives, UR: 1005-1718), and 13 times greater than the status quo (248 lives, UR: 179-316), resulting in an increment of 1962 (UR: 772-3153) and 3076 (UR: 1598-4555) deaths averted compared with the basic mCARE programme and the status quo. Based on the LiST model outputs, labour and delivery management was the major intervention saving lives (27\% for neonatal, $37 \%$ for maternal and $93 \%$ for stillbirth), and the major causes of deaths were sepsis for newborns $(41 \%)$, intrapartum for stillbirths $(97 \%)$ and hypertensive disorder for maternal lives $(46 \%)$.

\section{Incremental cost-effectiveness ratio}

We estimated the incremental cost-effectiveness of the comprehensive mCARE programme as $\$ 9684$ and $\$ 13979$ per death averted compared with the basic mCARE programme and the status quo, respectively (table 2). This translated into an estimated $\$ 327$ and $\$ 462$ per DALY averted compared with the basic mCARE programme and the status quo. With the continued implementation and geographical scaling-up, the model estimated that costeffectiveness of the mCARE programme may improve from $\$ 1152$ to $\$ 462$ per DALY averted over 5 to 10 years compared with the status quo.

\section{Sensitivity analyses}

In the one-way sensitivity analysis, the major drivers of cost-effectiveness were the number of lives saved for child 
Table 2 Summary of costs, health outcome and incremental cost-effectiveness ratios of the three scenarios: (1) comprehensive mCARE intervention package; (2) basic mCARE intervention package; and (3) paper-based status quo, over 2018-2027 in Bangladesh

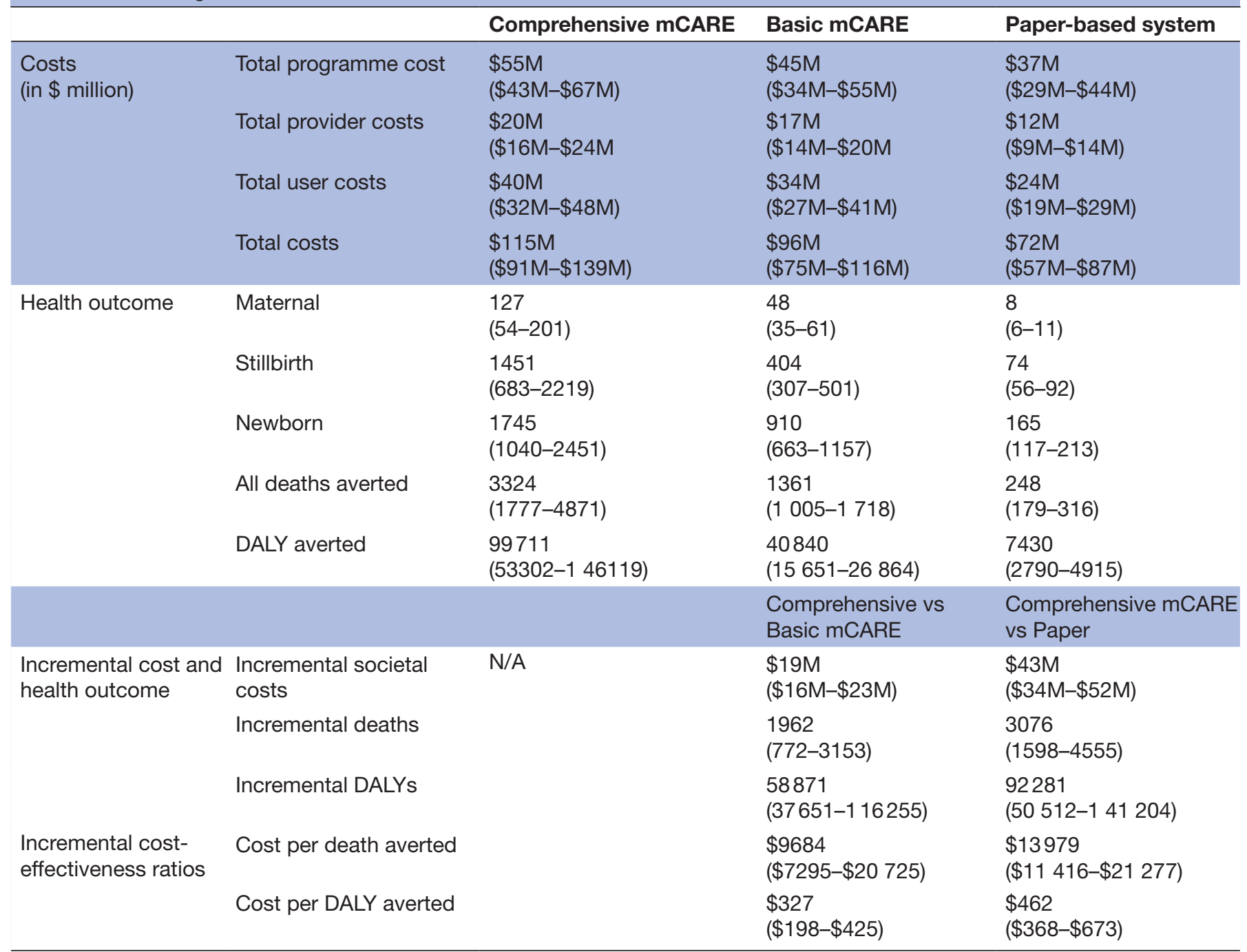

DALYs, disability-adjusted life years; NA, not applicable.

delivery between facility and community levels, followed by the programme costs and population coverage in the mCARE system (figure 1). Our model showed that the lower population and service coverage reduce the ICER (more cost-effective) as they influenced reducing the intervention costs more than deaths averted. In the three-way sensitivity analysis (figure 2), we found that the cost-effectiveness of mCARE programme can improve almost sevenfold from $\$ 1287$ to $\$ 172$ per DALY averted at child delivery at facility level, a lower mCARE programme cost and a lower population coverage (lower number of pregnancies registered, resulting in lower societal costs due to reduced care-seeking). The results of the PSA, displayed in the cost-effectiveness acceptability curves, show the probability of the intervention being costeffective under different willingness-to-pay thresholds (figure 3). At a priori willingness-to-pay threshold of $\$ 1000$ per DALY averted, for example, the comprehensive
mCARE programme was $50 \%$ cost-effective compared with the status quo over 5 years, $96 \%$ over 8 years and $100 \%$ over 10 years of implementation; at a willingnessto-pay threshold of $\$ 1700$ per DALY averted (Bangladesh GDP per capita in 2018), all scenarios were almost 100\% cost-effective relative to the status quo.

\section{DISCUSSION}

From a societal perspective, we estimated that the digital health intervention on pregnancy surveillance and careseeking promotion can be cost-effective relative to the paper-based status quo at $\$ 416$ per DALY averted with incremental geographical scale-up over 10 years (20182027) upto 10 million population in Bangladesh. Key drivers of cost-effectiveness were number of lives saved for child delivery between facility and community levels, followed by the programme costs and population coverage 


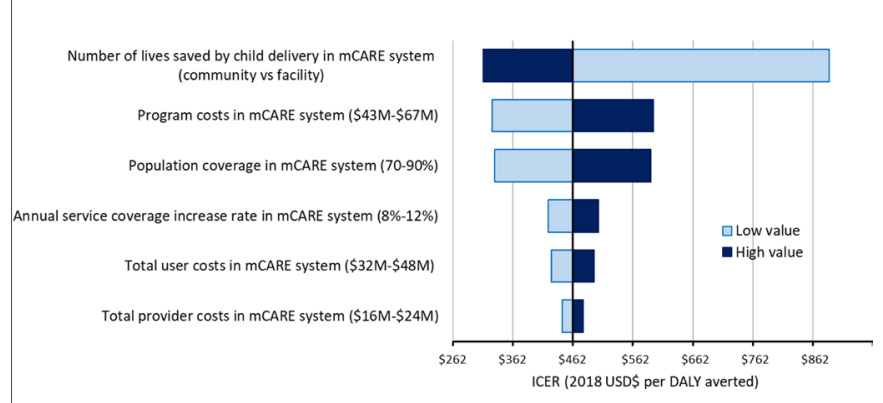

Figure 1 One-way sensitivity analysis of the costeffectiveness of comprehensive mCARE programme compared with the paper-based status quo in Bangladesh. The parameters shown had the greatest quantitative influence on the incremental cost-effectiveness of comprehensive mCARE programme relative to paper-based status quo in a one-way sensitivity analysis. The bars show the incremental cost-effectiveness (2018 US dollars per death averted) of the comprehensive mCARE group relative to the status quo under the high value (dark blue) and low value (light blue) of the parameter in question, holding all other parameters constant. For example, at a low health system capacity/ care-seeking scenario (ie, all incremental coverage increase of child delivery to be occurred by skilled health attendants in the home/community level rather than obstetric care clinics), the ICER increases from the base value of $\$ 462$ to $\$ 889$, suggesting that the mCARE programme becomes less cost-effective compared with the status quo. The vertical line corresponds to the reference scenario (values as in table 2, corresponding to $\$ 462$ per DALY averted). DALY, disabilityadjusted life year; ICER, incremental cost-effectiveness ratio.

in the mCARE system. The cost-effectiveness improves from $\$ 1152$ to $\$ 462$ per DALY averted from 5 to 10 years with the continued implementation and geographical scaling-up, despite of additional capital costs for population registration and training required.

Our study finding highlights that the cost-effectiveness of mHealth programmes are setting-specific and likely to change over time and scale. The three-way sensitivity analysis illustrates that cost-effectiveness of the mCARE system can be as low as $\$ 172$ per DALY averted in the settings (eg, urban) with high health system capacity and care-seeking for facility-based child deliveries, lower programme costs (due in part to high population density) and lower population coverage (due in part to wide-spread family planning programmes), compared with the settings in a rural/ hard-to-reach areas (\$1287 per DALY averted). In other words, while mCARE programme is expected to improve population and service coverage, the programme may end-up suboptimal cost-effectiveness with greater societal costs compared with the status quo, unless the increased care-seeking results in greater lives saving through highquality service especially at child delivery. Nevertheless, the extent of plausible ICER changes is still under Bangladesh GDP per capita (\$1700 in 2018), suggesting that mCARE programme would be considered cost-effective in a variety of health system settings.

\begin{tabular}{|c|c|c|c|c|}
\hline \multicolumn{5}{|c|}{ High health system capacity/care seeking level: child delivery at facility level } \\
\hline & & \multicolumn{3}{|c|}{ Population coverage } \\
\hline & & 0.70 & 0.81 (base case) & 0.90 \\
\hline \multirow{3}{*}{ Program cost } & $0.8(-20 \%)$ & $\$ 172$ & $\$ 221$ & $\$ 270$ \\
\hline & 1.0 (base case) & $\$ 263$ & $\$ 312$ & $\$ 361$ \\
\hline & $1.2(+20 \%)$ & $\$ 354$ & $\$ 403$ & $\$ 451$ \\
\hline \multicolumn{5}{|c|}{ Base case health system capacity/care seeking: child delivery at facility/community level } \\
\hline & & \multicolumn{3}{|c|}{ Population coverage } \\
\hline & & 0.70 & 0.81 (base case) & 0.90 \\
\hline \multirow{3}{*}{ Program cost } & $0.8(-20 \%)$ & $\$ 255$ & $\$ 327$ & $\$ 400$ \\
\hline & 1.0 (base case) & $\$ 389$ & $\$ 462$ & $\$ 534$ \\
\hline & $1.2(+20 \%)$ & $\$ 524$ & $\$ 596$ & $\$ 668$ \\
\hline \multicolumn{5}{|c|}{ Low health system capacity/care seeking: child delivery at community level } \\
\hline & & \multicolumn{3}{|c|}{ Population coverage } \\
\hline & & 0.70 & 0.81 (base case) & 0.90 \\
\hline \multirow{3}{*}{ Program cost } & $0.8(-20 \%)$ & $\$ 491$ & $\$ 630$ & $\$ 769$ \\
\hline & 1.0 (base case) & $\$ 750$ & $\$ 889$ & $\$ 1,028$ \\
\hline & $1.2(+20 \%)$ & $\$ 1,009$ & $\$ 1,148$ & $\$ 1,287$ \\
\hline
\end{tabular}

Figure 2 Three-way sensitivity analysis of the costeffectiveness of comprehensive mCARE programme compared with the paper-based status quo in Bangladesh. The heat map displays the incremental cost-effectiveness of the mCARE programme compared with the status quo, in units of cost per DALY averted. Each panel corresponds to a level (high, base case and low) of the number of lives saved by child delivery based on an assumption of different health system capacity/care-seeking level for child delivery from the Lives Saved Tool. A high health system capacity/ care-seeking scenario indicates that child delivery coverage increases at both community and facility levels from $42 \%$ and $38 \%$ to $65 \%$ and $58 \%$, respectively, over 2018-2027. A low health system capacity/care-seeking scenario indicates that child delivery coverage increases at both community from $42 \%$ to $65 \%$ and $38 \%$ for facility delivery throughout $2018-$ 2027. Each column represents a different level of population coverage (ie, number of pregnant women who were registered in the programme through census enumeration and pregnancy surveillance) and each row depicts a different level of programme costs (as a potential extent of economies of scale).

Compared with the previous studies on mHealth application (as a job aid for CHWs for pregnancy surveillance and health promotion for essential maternal and newborn healthcare services), our study shows a higher ICER estimate of $\$ 462$ per DALY averted from a societal perspective (from $\$ 1152$ to $\$ 462$ per DALY averted over 5 to 10 years) in Bangladesh. Prinja et at ${ }^{42}$ estimated the cost savings ( $\$ 90$ per DALY averted) from a societal perspective and $\$ 205$ per DALY averted over 10 years from a health system perspective in India, accounting a trade-off relationship between preventive and curative service use induced by mHealth intervention (ie, greater preventive care-seeking contributes to less illness and less curative care-seeking). Willcox et $a l^{14}$ and LeFevre et $a l^{13}$ estimated $\$ 174$ to $\$ 7$ per DALY averted in Ghana from 1 to 10 years from a programme perspective and $\$ 1985$ to $\$ 200$ per DALY averted in South Africa from 1 to 5 years from the societal perspective, with continued implementation and an increasing number of beneficiaries over time. Our ICER value is moderately higher than the previous findings as we incorporated a geographical expansion scenario which required additional capital cost investment in any new district. Furthermore, while we included considerable user costs incurred by increased 
A Cost-Effectiveness Acceptability Curves across the three scenarios

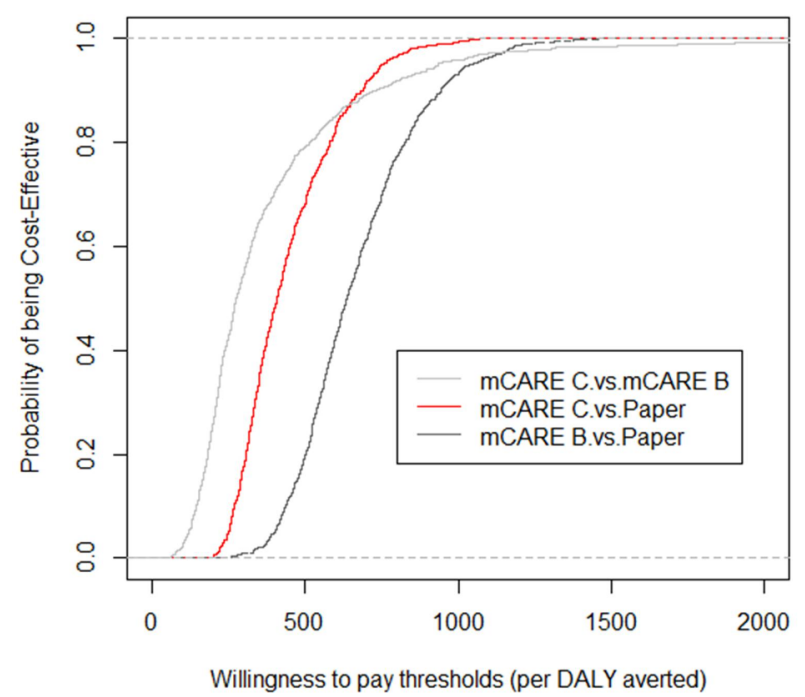

B Cost-Effectiveness Acceptability Curves over five to ten years of implementation: Comprehensive mCARE program vs Status quo

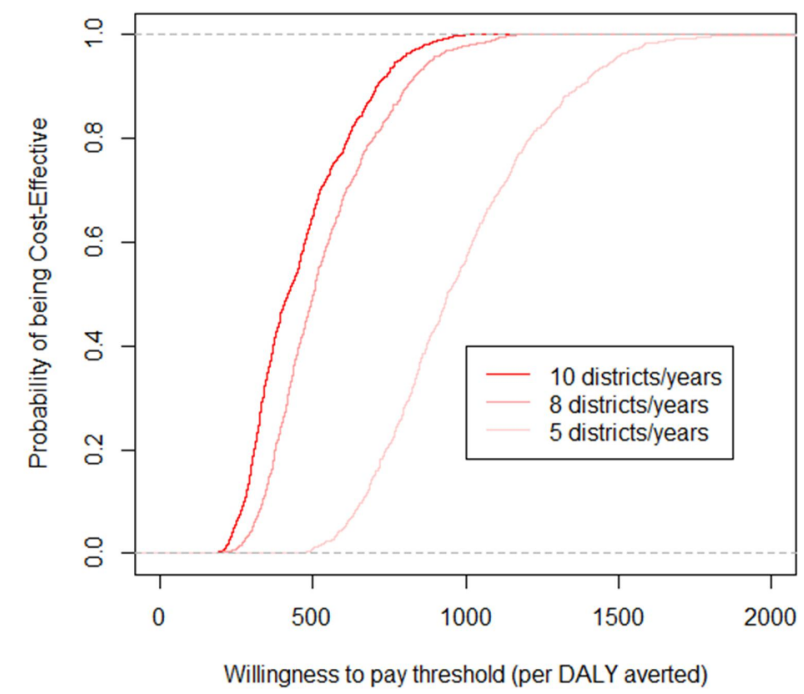

Figure 3 Cost-effectiveness acceptability curves of mCARE programmes compared with the paper-based status quo in Bangladesh. In this figure, the horizontal axis denotes the WTP threshold per DALY averted (ICER), and the vertical axis indicates the probability of intervention programme being cost-effective based on the proportion of simulations in which the comparison of two different programmes falls below the WTP threshold. In Panel A, the light grey line indicates the probabilities of being cost-effectiveness comparison of the comprehensive mCARE programme ('mCARE C') to the basic mCARE programme ('mCARE B'). The red line indicates the comparison of the comprehensive mCARE programme ('mCARE C') versus the paper programme ('paper'). The dark grey line indicates the comparison of the mCARE B versus paper programmes over 10 years (2018-2027) of implementation. In Panel B, each line indicates the probabilities of being cost-effectiveness comparison of mCARE C versus Paper in different implementation years. Costs are expressed in 2018 US dollars. DALY, disability-adjusted life year; ICER, incremental cost-effectiveness ratio; WTP, willingness-to-pay.

care-seeking by the intervention, we did not include lives saved for children older than 1 year in the LiST modelling. We also did not consider a potential trade-off relationship between prevention and curative care (which may decrease societal costs) due to limited data to define those distant and dynamic consequences.

Our model-based cost-effectiveness analysis shows a greater ICER ( $\$ 462$ vs $\$ 31$ per DALY averted), compared with the previous cost-effective analysis on the mCARE program $^{23}$ because of the different study perspectives, comparison groups, size of target population, and cost inputs and effectiveness estimations between the two studies. First, we compared mCARE system versus paperbased status quo (and thus incremental cost incurred from total mCARE programme costs as well as provider and user costs relative to paper-based status quo), while the previous study compared mCARE comprehensive versus basic programmes implemented from 2011 to 2015 in Gaibandha. Second, we constructed a hypothetical cohort and annual geographical expansion scenario upto 10 million population over 10 years (assuming 1 million population and 15000 pregnant women per district), while the previous study is based on 610 pregnant women enrolled from the observed data. All these contributed to far greater incremental costs and thus a higher ICER value in this model-based analysis compared with those from the previous study.

Our study has some limitations. First, there were limited empirical data and existing practice or evidence of a large scaled-up mHealth programme in pregnancy surveillance and care-seeking reminders. We therefore evaluated costeffectiveness with estimates drawn from observed data and a range of assumptions regarding the population and service coverage metrics using LiST to forecast health impact and costs. Future studies could also assess what specific aspects of scale-up (either geographical expansion vs sustainability) drives economies of scale more substantially to improve cost-effectiveness, whether or how nonlinear coverage increase to be observed by setting specific conditions, how or to what extent health system capacity and care-seeking influence service coverage and costs with empiric data and implementations, when approrpriate. Second, our model does not incorporate detailed complexities between preventive and curative care; while mCARE system may result in a more preventive care during pregnancy and thus less illness at delivery and less demand for curative care, the status quo with lower uptake of preventive care may result in more illness in the population and induce more demand for curative care. However, increased demand for curative care by sicker patients may avert fewer deaths than intended in the status quo. Overall, we think the trade-off relationship between preventive care-seeking and curative care has marginal effects on the incremental health outcome between the mCARE and status quo groups, and therefore may not change our final conclusion. Third, as many other studies use LiST, ${ }^{43-45}$ we linearly interpolated the level of service coverage from baseline to the target year 
for each scenario. Considering government-led scale-up in mind, our goal is to compare the relative impact of the mCARE programme to the paper-based status quo (rather to accurately project the coverage increase) under feasible scale-up implementation scenarios. Our findings thus intend to directly inform policy questions relating to determine the key drivers and enabling conditions to improve cost-effectiveness of a digital health programme in pregnancy surveillance and care-seeking promotion to help implementation planning and investment decision with pragmatic financial estimations. Nonetheless, it is important to acknowledge that the effectiveness of mHealth programmes is influenced by a series of settingspecific conditions, ${ }^{46}$ including literacy and education, geographical and financial access to health facilities, social and cultural norms, and women's access to information technology, number of available and trained CHWs and the quality or capacity of major MNH service provision agencies. ${ }^{47-49}$ Strength of our system and intervention strategy on this aspect is that the server system sends automated SMS to both pregnant women and CHWs and thus CHWs can make personal home visits to the pregnant women's houses to remind and check their ANC care-seeking. This feature allowed more equitable access to care as the pregnant women could receive care-sceeking stimuli by CHWs at least regardless of their mobile phone ownership and literacy or education levels.

Given that pregnancy surveillance and training are the major programme cost drivers, cost-sharing might also be a useful strategy to pursue with other public agencies and programmes, such as Civil Registration and Vital Statistics initiatives. We recognise that headcount-based denominators are rare in most low-resource health systems, despite the importance of a well-defined population to ensure 'universal' health coverage ${ }^{50}$ A well-established community-based pregnancy surveillance system using a mHealth platform linked to primary health service delivery can serve as a powerful source to create new incentives and value-added services for local businesses throughout the continuum of care among various health system stakeholders. Furthermore, as we found most lives saved occurred through effective intervention during child delivery, SMS and home-visit reminder intervention can be strategically focused on promoting child delivery at a facility level (eg, educating family members, and mobilising funds for child delivery). In parallel, as facilities and staffing are generally under-resourced in many developing countries, plans for scaling-up primary health services (eg, additional recruitment or training of CHWs, upgrading health facilities or health information systems) should be taken into account as it may be more important to ensure health system preparedness to meet the expected service needs and demands. ${ }^{51}$

\section{CONCLUSION}

In this study, we estimated that the digital health intervention on pregnancy surveillance and care-seeking promotion can be cost-effective relative to the paper-based status quo at $\$ 416$ per DALY averted with incremental geographical scale-up over 10 years upto 10 million pouplation in Bangladesh. While substantial initial capital costs for population registration and training may be necessary for any new district with incremental geographic expansion, continued implementation at scale may provide the potential to achieve cost and operational efficiency.

\section{Twitter Alain B Labrique @alabriqu}

Contributors $A B L$ is the principal investigator. $Y J, A E L$ and $A B L$ designed the study and data collection instruments. SM, HA, SS, RH, MC and KA oversaw the data collection and quantitative data entry. YJ trained a data collection team, analysed data and wrote the first draft of the manuscript. AEL, ABL and ESP provided comprehensive reviews and comments. All authors contributed and provided critical comments for the final manuscript.

Funding This work was supported by the UBS Optimus Foundation (Grant No. 11053414).

Competing interests None declared.

Patient consent for publication Not required.

Provenance and peer review Not commissioned; externally peer reviewed.

Data availability statement All data relevant to the study are included in the article or uploaded as supplemental information.

Supplemental material This content has been supplied by the author(s). It has not been vetted by BMJ Publishing Group Limited (BMJ) and may not have been peer-reviewed. Any opinions or recommendations discussed are solely those of the author(s) and are not endorsed by BMJ. BMJ disclaims all liability and responsibility arising from any reliance placed on the content. Where the content includes any translated material, BMJ does not warrant the accuracy and reliability of the translations (including but not limited to local regulations, clinical guidelines, terminology, drug names and drug dosages), and is not responsible for any error and/or omissions arising from translation and adaptation or otherwise.

Open access This is an open access article distributed in accordance with the Creative Commons Attribution Non Commercial (CC BY-NC 4.0) license, which permits others to distribute, remix, adapt, build upon this work non-commercially, and license their derivative works on different terms, provided the original work is properly cited, appropriate credit is given, any changes made indicated, and the use is non-commercial. See: http://creativecommons.org/licenses/by-nc/4.0/.

\section{ORCID iDs}

Youngji Jo http://orcid.org/0000-0002-1440-2786

Amnesty Elizabeth LeFevre http://orcid.org/0000-0001-8437-7240

Kelsey Alland http://orcid.org/0000-0001-8464-6308

\section{REFERENCES}

1 World Health Organization. Bangladesh. Available: https://www.who. int/docs/default-source/country-profiles/bangladesh.pdf?sfvrsn= 3c5a0134_6 [Accessed 5 Jan 2020].

2 UNICEF. Maternal and newborn health disparities Bangladesh Bangladesh. Available: https://data.unicef.org/wp-content/uploads/ country_profiles/Bangladesh/country\%20profile_BGD.pdf [Accessed 5 Jan 2020].

3 Halim A, Aminu M, Dewez JE, et al. Stillbirth surveillance and review in rural districts in Bangladesh. BMC Pregnancy Childbirth 2018;18:224.

4 USAID. Multi-sectoral nutrition strategy. 2014-2025. Available: https://www.usaid.gov/sites/default/files/documents/1864/1000days-brief-508.pdf [Accessed 5 Jan 2020].

5 El Arifeen S, Christou A, Reichenbach L, et al. Community-based approaches and partnerships: innovations in health-service delivery in Bangladesh. Lancet 2013;382:2012-26.

6 World Health Orgnization. Success Factors for Women's and Children's Health. Bangladesh. Available: http://www.who.int/pmnch/ knowledge/publications/bangladesh.pdf [Accessed 5 Jan 2020].

7 World Health Orgnization. mHealth: New horizons for health through mobile technologies, 2011. Available: balhealthaction.net/index.php/ gha/article/view/31473 
8 Little A, Medhanyie A, Yebyo $\mathrm{H}$, et al. Meeting community health worker needs for maternal health care service delivery using appropriate mobile technologies in Ethiopia. PLoS One 2013;8:e77563.

9 Macleod B, Phillips J, Stone AE, et al. The architecture of a software system for supporting community-based primary health care with mobile technology: the mobile technology for community health (MoTeCH) initiative in Ghana. Online J Public Health Inform 2012;4. doi:10.5210/ojphi.v4i1.3910. [Epub ahead of print: 17 May 2012].

10 Free C, Phillips G, Watson L, et al. The effectiveness of mobile-health technologies to improve health care service delivery processes: a systematic review and meta-analysis. PLoS Med 2013;10:e1001363.

11 Free C, Phillips G, Galli L, et al. The effectiveness of mobile-health technology-based health behaviour change or disease management interventions for health care consumers: a systematic review. PLoS Med 2013;10:e1001362

12 Datta SS, Ranganathan P, Sivakumar KS. A study to assess the feasibility of text messaging service in delivering maternal and child healthcare messages in a rural area of Tamil Nadu, India. Australas Med J 2014;7:175-80.

13 LeFevre A, Cabrera-Escobar MA, Mohan D, et al. Forecasting the value for money of mobile maternal health information messages on improving utilization of maternal and child health services in Gauteng, South Africa: cost-effectiveness analysis. JMIR Mhealth Uhealth 2018;6:e153.

14 Willcox M, Moorthy A, Mohan D, et al. Mobile technology for community health in Ghana: is maternal messaging and provider use of technology cost-effective in improving maternal and child health outcomes at scale? J Med Internet Res 2019;21:e11268.

15 Källander K, Tibenderana JK, Akpogheneta OJ, et al. Mobile health (mHealth) approaches and lessons for increased performance and retention of community health workers in low- and middle-income countries: a review. J Med Internet Res 2013;15:e17.

16 Fottrell E, Ahmed N, Morrison J, et al. Community groups or mobile phone messaging to prevent and control type 2 diabetes and intermediate hyperglycaemia in Bangladesh (DMagic): a cluster-randomised controlled trial. Lancet Diabetes Endocrinol 2019;7:200-12.

17 Ahmed T, Lucas $\mathrm{H}$, Khan AS, et al. eHealth and mHealth initiatives in Bangladesh: a scoping study. BMC Health Serv Res 2014;14:260.

18 World Health Orgnization. mHealth: new horizons for health through mobile technologies, 2011. Available: balhealthaction.net/index.php/ gha/article/view/31473

19 Kahn JG, Jiwani A, Gomez GB, et al. The cost and cost-effectiveness of scaling up screening and treatment of syphilis in pregnancy: a model. PLoS One 2014;9:e87510.

20 Aranda-Jan CB, Mohutsiwa-Dibe N, Loukanova S. Systematic review on what works, what does not work and why of implementation of mobile health (mHealth) projects in Africa. BMC Public Health 2014;14:188.

21 Eysenbach G, CONSORT-EHEALTH Group. CONSORT-EHEALTH: improving and standardizing evaluation reports of web-based and mobile health interventions. J Med Internet Res 2011;13:e126.

22 World Health Organization, Johns Hopkins University-Global mHealth Initiative, United Nations Foundation. The maps toolkit: $\mathrm{mHealth}$ assessment and planning for scale, 2015.

23 Jo Y, LeFevre AE, Healy K, et al. Costs and cost-effectiveness analyse of mCARE strategies for promoting care seeking of maternal and newborn health services in rural Bangladesh. PLoS One 2019;14:e0223004.

24 USAID. Bangladesh demographic and health survey (BDHS), 2014. Available: https://dhsprogram.com/publications/publication-fr311dhs-final-reports.cfm [Accessed 5 Jan 2020].

25 BRAC. Gaibandha. Available: https://brac.net/images/Gaibandha.pdf [Accessed 5 Jan 2020].

26 World Bank Data. Bangladesh. Available: https://data.worldbank.org/ indicator/SE.PRM.CMPT.ZS?locations=BD\&view=chart [Accessed 5 Jan 2020].

27 Centers for Disease Control and Prevention (CDC): Division of Reproductive Health. Estimating the number of pregnant women in a geographic area.

28 Bangladesh Bureau of statistics. Available: http://203.112.218.65:8008/ [Accessed 5 Jan 2020].

29 GBD 2017 Population and Fertility Collaborators. Population and fertility by age and sex for 195 countries and territories, 1950-2017: a systematic analysis for the global burden of disease study 2017. Lancet 2018;392:1995-2051.

30 Benson J, Andersen K, Samandari G. Reductions in abortion-related mortality following policy reform: evidence from Romania, South Africa and Bangladesh. Reprod Health 2011;8:39.

31 Abir T, Agho KE, Ogbo FA, et al. Predictors of stillbirths in Bangladesh: evidence from the 2004-2014 nation-wide household surveys. Glob Health Action 2017;10:1410048.

32 Singh N, Labrique A, Mehra S. Can an integrated mHealth system increase utilization of antenatal care services and promote safe delivery? 2014.

33 Lives Saved Tool. Available: https://www.livessavedtool.org/ [Accessed 5 Jan 2020].

34 LeFevre AE, Shillcutt SD, Waters HR, et al. Economic evaluation of neonatal care packages in a cluster-randomized controlled trial in Sylhet, Bangladesh. Bull World Health Organ 2013;91:736-45.

35 Pitt C, Tawiah T, Soremekun S, et al. Cost and cost-effectiveness of newborn home visits: findings from the Newhints clusterrandomised controlled trial in rural Ghana. Lancet Glob Health 2016;4:e45-56.

36 World Health Orgnization. Life tables by country Bangladesh. Available: http://apps.who.int/gho/data/view.main.60120?lang=en [Accessed 5 Jan 2020].

37 Shillcutt SD, Lefevre AE, Lee ACC, et al. Forecasting burden of longterm disability from neonatal conditions: results from the Projahnmo I trial, Sylhet, Bangladesh. Health Policy Plan 2013;28:435-52.

38 USAID. Measurement and accountability for health in Bangladesh a status report, 2016. Available: https://www.measureevaluation.org/ resources/publications/tr-16-130 [Accessed 5 Jan 2020].

39 International monetary fund: Bangladesh. Available: http://www.imf. org/external/country/ [Accessed 5 Jan 2020].

40 Jo Y, Alland K, Ali H, et al. Antenatal care in rural Bangladesh: current state of costs, content and recommendations for effective service delivery. BMC Health Serv Res 2019:19:861.

41 Marseille E, Larson B, Kazi DS, et al. Thresholds for the costeffectiveness of interventions: alternative approaches. Bull World Health Organ 2015;93:118-24.

42 Prinja S, Bahuguna P, Gupta A, et al. Cost effectiveness of mHealth intervention by community health workers for reducing maternal and newborn mortality in rural Uttar Pradesh, India. Cost Eff Resour Alloc 2018;16:25

43 Roberton T, Carter ED, Chou VB, et al. Early estimates of the indirect effects of the COVID-19 pandemic on maternal and child mortality in low-income and middle-income countries: a modelling study. Lancet Glob Health 2020;8:e901-8.

44 Nove A, Friberg IK, de Bernis L, et al. Potential impact of midwives in preventing and reducing maternal and neonatal mortality and stillbirths: a lives saved tool modelling study. Lancet Glob Health 2021;9:e24-32.

45 Chou VB, Walker N, Kanyangarara M. Estimating the global impact of poor quality of care on maternal and neonatal outcomes in 81 low- and middle-income countries: a modeling study. PLOS Med 2019:16:e1002990.

46 Mangham-Jefferies L, Pitt C, Cousens S, et al. Erratum: costeffectiveness of strategies to improve the utilization and provision of maternal and newborn health care in low-income and lower-middleincome countries: a systematic review. BMC Pregnancy Childbirth 2015;15:64

47 Hafkin N. Gender issues in ICT policy in developing countries: an overview. United Nations Division for the Advancement of Women (DAW), 2002.

48 LeFevre AE, Mohan D, Hutchful D, et al. Mobile technology for community health in Ghana: what happens when technical functionality threatens the effectiveness of digital health programs? BMC Med Inform Decis Mak 2017;17:27.

49 Labrique A, Vasudevan L, Weiss W, et al. Establishing standards to evaluate the impact of integrating digital health into health systems. Glob Health Sci Pract 2018;6:S5-17.

50 Storisteanu DML, Norman TL, Grigore A, et al. Can biometrics beat the developing world's challenges? Biometric Technology Today 2016;2016:5-9.

51 Collins DH, Jarrah Z, Ndizeye C, et al. The cost of scaling up primary health-care services-comparisons from studies in six countries: economic research using systematic sampling. The Lancet 2013;381:S30. 